\title{
Development of Endoscopic Diagnosis and Treatment for Chronic Unilateral Hematuria: 35 Years Experience
}

\author{
Ryuta Tanimoto, MD, $\mathrm{PhD}{ }^{1,2}$ Hiromi Kumon, $\mathrm{MD}, \mathrm{PhD}^{1,3}$ and Demetrius $\mathrm{H}$. Bagley, $\mathrm{MD}^{2}$
}

\begin{abstract}
Purpose: Chronic unilateral hematuria (CUH) is also called lateralizing essential hematuria, benign essential hematuria, and benign lateralizing hematuria, which was defined as intermittent or continuous gross hematuria that cannot be diagnosed with standard radiology and hematology studies, together with unilateral bloody efflux by cystoscopy. CUH is rare, but sometimes confused with malignancy or life-threatening hemorrhage. Therefore, it can cause considerable anxiety to not only patients but also urologists. For this study, we summarized articles about endoscopic diagnosis and treatment of $\mathrm{CUH}$, and discussed the development of endourology for CUH.

Materials and Methods: We searched articles related to CUH that were indexed in the PubMed database and published in English. Key terms used were "unilateral," "lateralizing," "chronic," "benign," and "idiopathic" hematuria. We found 15 pertinent articles reporting CUH.

Results: Endoscopically, CUH can be classified into three categories: discrete lesion, diffuse lesion, or no (unidentified) lesion. Currently, endoscopic techniques for CUH are similar to the techniques for upper tract urothelial carcinoma, using semi-rigid and flexible ureteroscopes with diathermy fulguration or laser ablation for treatment. The overall success rate of endoscopic treatment for $\mathrm{CUH}$, defined as resolution of gross hematuria after treatment, was 93\% (190/205). The recurrence rate, defined as recurrent gross hematuria after treatment, was $10 \%(19 / 189)$.

Conclusion: Advancements in endoscopic devices and techniques have enabled more accurate and less invasive diagnosis and treatment of $\mathrm{CUH}$. Once $\mathrm{CUH}$ is defined, flexible ureteroscopy is the diagnostic and therapeutic technique of choice.
\end{abstract}

Keywords: chronic unilateral hematuria, flexible ureteroscopy, semi-rigid ureteroscopy

\section{Introduction}

A MONG PATIENTS WITH gross hematuria there are some patients in whom imaging studies and urine cytology are normal and the hematuria clearly is seen to originate from one ureteral orifice at cystoscopy. Such patients often seek additional opinions and undergo repeated and unhelpful investigations. Even though further workup including second line urine microscopy, clotting/coagulopathy studies, renal biopsy, hemoglobin electrophoresis, and renal angiography are often undertaken, the diagnosis may remain unidentified. These conditions have been recognized as unilateral benign hematuria, lateralizing essential hematuria, or chronic unilateral hematuria (CUH).

In this review, we summarize the etiology and endoscopic diagnosis and treatment of CUH.

\section{Materials and Methods}

We searched articles related to CUH that were indexed in the PubMed database and published in English. We did not define a restricted range for publication date. Key terms used were "unilateral" or "lateralizing hematuria" or "chronic," "benign," or "idiopathic hematuria." We excluded articles in which patients were treated by nonendoscopic modalities, such as nephrectomy or medical treatment.

\section{Results}

We found 15 pertinent articles including 288 cases of $\mathrm{CUH}$ in PubMed database. ${ }^{1-15}$ Endoscopically, CUH can be classified into three categories: discrete lesion, diffuse lesion, or no (unidentified) lesion (Table 1).

\footnotetext{
${ }^{1}$ Department of Urology, Okayama University Hospital, Okayama, Japan.

${ }^{2}$ Department of Urology, Thomas Jefferson University Hospital, Philadelphia, Pennsylvania.

${ }^{3}$ Niimi College, Niimi, Okayama, Japan.
} 
Table 1. Etiology of Chronic Unilateral Hematuria

\begin{tabular}{|c|c|c|c|c|c|c|c|c|c|c|c|c|c|c|}
\hline Year & First author & Total & Hemangioma & $\begin{array}{l}\text { Minute } \\
\text { venous } \\
\text { rupture }\end{array}$ & Calculi & $T C C$ & $\begin{array}{c}\text { Focal } \\
\text { erythema }\end{array}$ & $\begin{array}{c}\text { Not } \\
\text { specified }\end{array}$ & $\begin{array}{c}\text { Discrete } \\
\text { lesion }\end{array}$ & $\%$ & $\begin{array}{c}\text { Diffuse } \\
\text { lesion }\end{array}$ & $\%$ & $\begin{array}{c}\text { No } \\
\text { lesion }\end{array}$ & $\%$ \\
\hline 2014 & Takazawa & 7 & 3 & 4 & & & & & 7 & 100 & 0 & 0 & 0 & 0 \\
\hline 2012 & Araki & 104 & 21 & 64 & 1 & & & & 86 & 83 & 0 & 0 & 18 & 17 \\
\hline 2009 & Brito & 13 & 9 & 4 & & & & & 13 & 100 & 0 & 0 & 0 & 0 \\
\hline 2007 & Mugiya & 23 & 2 & 14 & 2 & & & & 18 & 78 & 0 & 0 & 5 & 22 \\
\hline 1999 & Yazaki & 15 & 1 & 2 & & 4 & 1 & 2 & 10 & 67 & 0 & 0 & 5 & 33 \\
\hline 1999 & Mugiya & 4 & 1 & 3 & & & & & 4 & 100 & 0 & 0 & 0 & 0 \\
\hline 1998 & Tawfiek & 23 & 7 & 3 & 2 & 3 & & & 15 & 65 & 3 & 13 & 5 & 22 \\
\hline 1997 & Nakada & 17 & 5 & 0 & 1 & 1 & 5 & & 12 & 71 & 2 & 12 & 3 & 18 \\
\hline 1994 & Desgrandchamps & 8 & & 1 & & & 2 & & 3 & 38 & 4 & 50 & 1 & 13 \\
\hline 1990 & Bagley & 30 & 11 & 3 & 1 & 1 & & & 16 & 53 & 9 & 30 & 5 & 17 \\
\hline 1990 & Kumon & 12 & 4 & 4 & & 1 & & & 9 & 75 & 1 & 8 & 2 & 17 \\
\hline 1989 & Kavoussi & 8 & & & & & & 6 & 6 & 75 & 0 & 0 & 2 & 25 \\
\hline 1987 & McMurty & 8 & & 4 & 1 & & & & 5 & 63 & 3 & 38 & 0 & 0 \\
\hline 1984 & Patterson & 4 & & 2 & & & & & 2 & 50 & 2 & 50 & 0 & 0 \\
\hline \multirow{2}{*}{1981} & Gittes & 12 & 5 & & & & & & 5 & 42 & 7 & 58 & 0 & 0 \\
\hline & Total & 288 & 69 & 108 & 8 & 10 & 8 & 8 & 211 & 73 & 31 & 11 & 46 & 16 \\
\hline
\end{tabular}

In the discrete lesion category, renal hemangioma, minute venous rupture (MVR), and other intraluminal lesions were identified. Renal hemangiomas can range in size from nearly microscopic elevations or red spots to larger vascular appearing lesions up to several millimeters in diameter. ${ }^{16}$ They can be usually found in the papillary tips. MVR is characterized as small lesions that result from the fragile vasculatures in renal papilla or fornix. ${ }^{17}$ Peripapillary varices are most likely in the same category as MVRs. Other intraluminal lesions include upper urinary tract urothelial cancers and small calculi that are not detected by standard urologic evaluation, such as noncontrast CT, CT urography, or voided urine cytology.

The overall success rate of endoscopic treatment for unilateral hematuria, defined as resolution of gross hematuria after endoscopic treatment, was 93\% (190/205). When limited to discrete lesions, the rate was $95 \%$ (71/75), while the success rate for diffuse lesions was $69 \%$ (9/13). When no clear abnormality can be detected, the success rate was $71 \%$ $(10 / 14)$ even if untreated, as shown in Table 2.

The recurrence rate, defined as recurrent gross hematuria after treatment in those who had been followed, was $10 \%$ (19/189). Araki et al. reported seven cases of recurrent gross hematuria in their results over a 22-year experience with median follow-up of 139 months. Only one patient required ureteroscopy for hemostasis, but another six patients stopped bleeding spontaneously. ${ }^{14}$ Nakada et al. also reported seven cases of recurrence in median follow-up of 58 months, with most patients having recurrence within 6 months. Of these patients, all three patients with treated diffuse lesions and two out of three patients who had a negative examination had recurrent bleeding. ${ }^{8}$

\section{Discussion}

CUH is characterized by intermittent or continuous gross hematuria that cannot be diagnosed with standard radiologic and hematologic studies. Cystoscopic examination must define that the hematuria originates from unilateral upper urinary tract. Although most cases have no complications in their natural course, massive hematuria can rarely cause anemia and can be associated with significant renal colic with the passage of clots. ${ }^{2}$

Historically, patients with CUH have been managed conservatively by bed rest, blood transfusion, or oral administration of hemostatic medication such as carbazochrome sodium sulfonate, tranexamic acid, aminocaproic acid, or instillation of silver nitrate into the renal pelvis. Although these medications have been established for refractory hematuria in the lower urinary tract, only few data were reported for the upper urinary tract. ${ }^{18-22}$ Nash and Henry reported a case in which severe unilateral essential hematuria was well treated with amonocaproic acid, but they warned its use should be discreet because it could cause microangiopathic thrombosis or obstruction of the urinary tract due to clot formation. ${ }^{23}$ In Araki's article, the authors showed instillation of silver nitrate into renal pelvis before ureteroscopy was less frequent and administration of hemostatic medication was no more than supportive for $\mathrm{CUH} .{ }^{14}$ As a last resort for managing intractable hematuria, some patients underwent nephrectomy. ${ }^{23,24}$

Newer technologies have allowed more accurate and less invasive diagnosis and treatment for CUH.

First development for the endoscopic evaluation of $\mathrm{CUH}$ was the nephroscope. In 1981, Gittes and Varady ${ }^{1}$ applied the technique of intraoperative nephroscopy in patients with $\mathrm{CUH}$ to guide their surgical therapy. A nephroscope was inserted into the renal pelvis through a small pyelotomy to visualize the collecting system. Bleeding sites could be identified in all patients. This is noteworthy that they first utilized endoscopy to diagnose CUH. The lesions were treated by partial nephrectomy in 12 patients and nephrectomy in 1 . In seven of the patients treated by partial nephrectomy, no abnormalities were found, while discrete submucosal hemangiomas were found in five cases.

The second development was the appearance of rigid ureteroscopy. Patterson and colleagues ${ }^{2}$ demonstrated full endoscopic evaluation and management of unilateral hematuria using both rigid ureteroscopes and nephroscopes. They tried to evaluate upper urinary tract with rigid ureteroscopy, by 


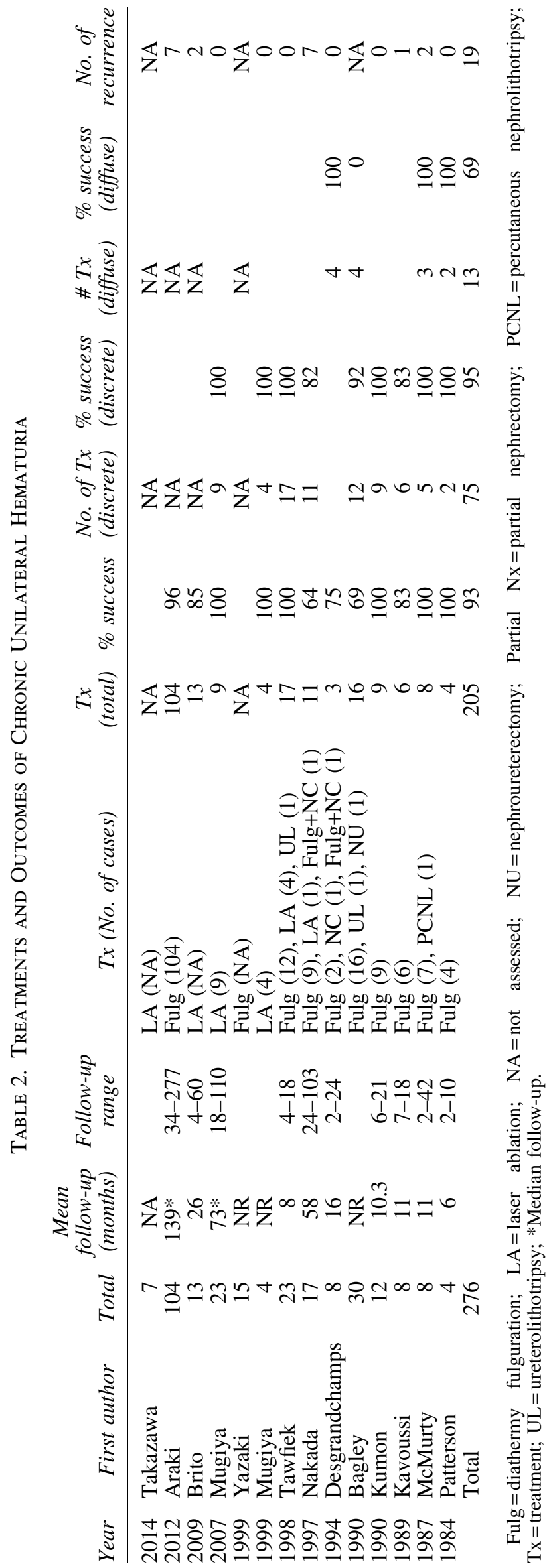

which the proximal ureter and renal collecting system might not be visualized, especially in men. If this did not identify a specific source of bleeding or if the source was not manageable, then percutaneous access was performed. Development of percutaneous access to the collecting system and availability of nephroscopes had made endoscopic evaluation of the renal collecting system a reliable and practical technique. However, percutaneous access required two stages: lithotomy position for retrograde access and prone position for anterograde access. Furthermore, this might conceal the bleeding site where access was made and induce bleeding to obscure vision.

The third development was a flexible deflectable ureteroscope. McMurty and colleagues ${ }^{3}$ corroborated and expanded on the above report but used flexible deflectable ureteroscopy in one patient to treat without percutaneous access. Later, a combination of a flexible ureteroscope (FU) and semi-rigid ureteroscope became the standard technique. Kavoussi and colleagues ${ }^{4}$ reported on their diagnostic and therapeutic experience with flexible deflectable ureteroscopy in 68 patients, including 8 patients with unilateral hematuria. Using retrograde access with flexible and rigid ureteroscopes, they were successful in accessing the area of interest in $93 \%$ of the patients. Complete visualization of the entire collecting system was possible in only $65 \%$ of the patients in whom it was attempted. However, greater than $75 \%$ of the calices could be examined in all patients except two who had infundibular stenosis.

Kumon and colleagues ${ }^{5}$ reported on 12 patients with unilateral hematuria by rigid and flexible ureteroscopy including utilization of conventional percutaneous pyeloscopy for the initial two patients. They observed the entire collecting system in all 12 patients and performed cold cup biopsies in 6 patients to confirm the histology. In this report, they first described "MVR," identified as bleeding from small dilated veins on renal papillae or rupture of bridging vein between renal papillae and calyceal fornix. Retrospectively, former authors had similar findings. Bagley and Allen ${ }^{6}$ also reported on 32 patients with benign essential hematuria using actively deflectable ureteroscopes, which allowed examination of the entire intrarenal collecting system in 28 of the 32 patients. The mid ureter could not be passed with the specific instrument available in two patients early in their series, while in two other patients the lower infundibula could not be inspected.

In this report, four cases had abnormalities located throughout the intrarenal collecting system that they unsuccessfully attempted to treat with fulguration. They suggested that these diffuse abnormalities were either not responsible for the bleeding, or in fact represented a more extensive process that allowed persistent bleeding in untreated areas. ${ }^{6}$ On the other hand, Desgrandchamps et al. reported three out of four successful treatments of diffuse lesions. ${ }^{7}$ They considered these lesions as nonspecific pyelitis based on the histology, which showed simple edema of the lamina propria with no vascular anomalies. ${ }^{7}$ Although the etiology of diffuse lesions remains unclear, the rate of diffuse lesion has decreased over time and no cases have been reported since 1999. This might have resulted from advancements in imaging and endoscopic techniques.

Fourth development was digital endoscope. Takazawa and collagues reported seven cases of CUH with a digital FU, URF-V (Olympus, Tokyo, Japan), with an 8.5F tip diameter and $9.9 \mathrm{~F}$ shaft diameter, which had the CCD imaging sensor placed at the distal end of the ureteroscope. This provided 
moiré-free, bright images and made it lighter because of no camera head attachment. They inserted the FU through a ureteral access sheath and systematically evaluated the upper urinary tract. ${ }^{15}$ Latest models using tiny complementary metal-oxide-semiconductor (CMOS) chip are $8.5 \mathrm{~F}$ of shaft and tip diameter so that digital ureteroscopy is more useful for the diagnosis and treatment of $\mathrm{CUH}$.

As ureteroscopic devices have been miniaturized and developed more precise endoscopic techniques have been established. Fundamentally, endoscopic techniques for CUH were similar to the techniques for upper urinary tract urothelial cancer. ${ }^{25}$ After examining the lower urinary tract, the upper urinary tract should be examined directly without any iatrogenic injury, so-called "no touch techniques." A 6.9F semi-rigid ureteroscope is inserted into the bladder and introduced into the ureteral orifice under direct vision. ${ }^{26,27}$ The distal ureter, which may have some lesions causing CUH, should be evaluated before any instrumentation such as guidewire. Another advantage of using small caliber semirigid ureteroscope is gradual calibration of distal ureter because of tapered scope whose tip size is $6.9 \mathrm{~F}$ and proximal shaft size is $10.2 \mathrm{~F}$. Once the scope passes beyond the level of the iliac vessels, a guidewire is left at the level we have inspected and the semi-rigid ureteroscope is removed. A FU is inserted over the wire under fluoroscopic guidance. It is important not to allow the guidewire to migrate proximally, which may cause urothelial injury. The FU is then advanced under direct vision to examine the whole ureter until the renal pelvis.

In another no touch technique, the FU is inserted through the urethra and passed into ureteral orifice directly, eliminating the need for semi-rigid ureteroscope or guidewire. Due to miniaturization of ureteroscopes, it is rarely necessary to routinely dilate the ureter or the ureteral orifice, which might traumatize it. Although a no touch technique is always attempted, a guidewire should be utilized anytime it appears difficult to access the ureter safely. Another technical point is irrigation. Higher intraluminal pressure during ureteroscopy may obscure minute venous bleeding. Therefore, gentle irrigation is critical in this procedure. In most reports, irrigation was maintained by gravity and/or manual power irrigation. Araki et al. reported use of a monitored irrigation pressure system to maintain pressure at $40 \mathrm{mmHg}$, by which they identified significantly more MVR and fewer unidentified lesions. ${ }^{14}$

Because of miniaturization of current ureteroscopes, instruments through these ureteroscopes are limited to $3 \mathrm{~F}$. Diathermy fulguration has been used in $>80 \%$ of cases because it is safe, inexpensive, widely available, and established in the treatment of bladder lesions. Holmium:yttrium aluminium garnet (YAG) or Neodymium:YAG laser treatment has also been used. The advantage of laser treatment is the ability to use it in normal saline instead of water. Holmium:YAG penetrates tissues $<0.5 \mathrm{~mm}$ so that the target area can be more focused, while Neodymium:YAG has higher affinity for hemoglobin and penetrates deeper so that it results in more effective hemostasis. The disadvantages of laser treatments are (1) higher cost and (2) greater rigidity than a $2 \mathrm{~F}$ electrode, which may prevent full deflection.

We propose an algorithm for management of unilateral hematuria as simple and efficient as possible (Fig. 1). The diagnosis of CUH is made by patient history and physical exam, urinalysis, urine cytology, blood test including BUN

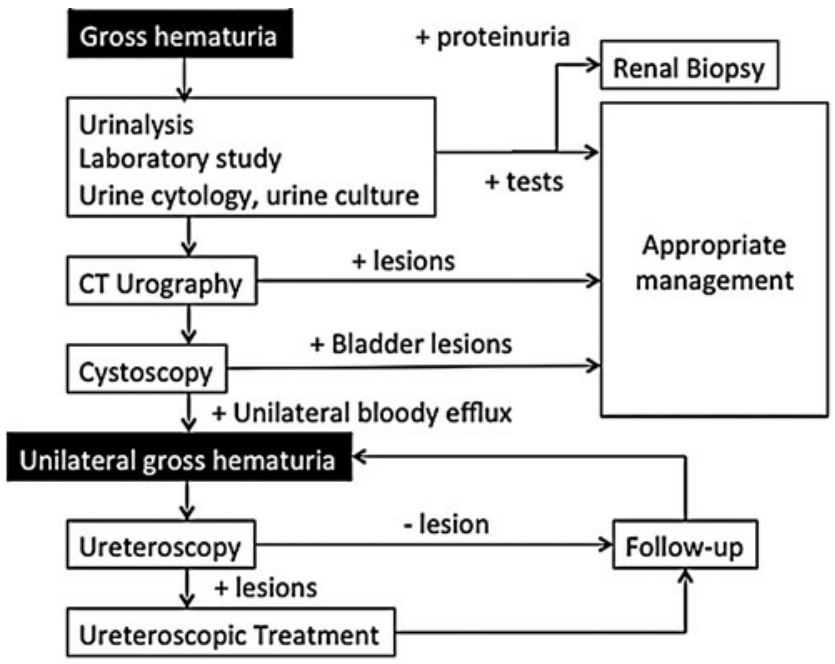

FIG. 1. Algorithm for management of chronic unilateral hematuria.

and creatinine, CBC with differential, PT, PTT, upper tract imaging that fails to identify any source of bleeding, and cystoscopy that identifies a unilateral bloody efflux. Patients with gross hematuria must undergo work up to rule out urological malignancy. According to AUA guidelines, cystoscopy and urine cytology should be considered for patients with gross hematuria because they have a higher risk of malignancy. CT urogram (CTU) is preferred over intravenous urogram (IVU) for hematuria because it has a higher sensitivity and specificity for detecting upper tract pathology than IVU. ${ }^{28-31}$ When CTU is not available, a combination of IVU and renal ultrasound is an alternative. Once unilateral hematuria is diagnosed by cystoscopy, patients should have diagnostic ureteroscopy as described above.

There is no consensus for a follow-up schedule after ureteroscopic treatment. Brito and colleagues followed up patients with urinalysis at $1,3,6$, and 12 months, and then annually after treatment. ${ }^{13}$ Araki and colleagues reported follow-up including urinalysis, urine cytology, and ureteroscopy every 3 months in the first 2 years, every 6 months in years 3 to 5 , and annually thereafter in cases of malignancy. ${ }^{14}$ However, the overall recurrence rate was quite low, 10\% (19/ 189), and even if gross hematuria recurred, it was well treated or exhibited negative bleeding during ureteroscopy. Therefore, once malignancy has been absolutely ruled out, ureteroscopy should be delayed until gross hematuria recurs.

\section{Conclusion}

CUH used to be difficult to diagnose and treat. The smaller semi-rigid and FUs have enabled near 100\% access to the entire collecting system. Newer digital ureteroscopes may enable more accurate diagnosis. The smaller working devices have allowed more accurate treatment of target lesions. Once CUH is defined, flexible ureteroscopy is the diagnostic and therapeutic technique of choice.

\section{Author Disclosure Statement}

Dr. Bagley is a consultant for Bard, Cook, and Olympus/ ACMI. For remaining authors, no competing financial interests exist. 


\section{References}

1. Gittes RF, Varady S. Nephroscopy in chronic unilateral hematuria. J Urol 1981;126:297-300.

2. Patterson DE, Segura JW, Benson RC, Jr., Leroy AJ, Wagoner R. Endoscopic evaluation and treatment of patients with idiopathic gross hematuria. J Urol 1984;132:1199-1200.

3. McMurty JM, Clayman RV, Preminger GM. Endourologic diagnosis and treatment of essential hematuria. J Endourol 1987;1:145-151.

4. Kavoussi L, Clayman RV, Basler J. Flexible, actively deflectable fiberoptic ureteronephroscopy. J Urol 1989;142: 949-954.

5. Kumon H, Tsugawa M, Matsumura Y, Ohmori H. Endoscopic diagnosis and treatment of chronic unilateral hematuria of uncertain etiology. J Urol 1990;143:554-558.

6. Bagley DH, Allen J. Flexible ureteropyeloscopy in the diagnosis of benign essential hematuria. J Urol 1990;143:549-553.

7. Desgrandchamps F, Piergiovanni M, Cussenot O, et al. Exploration and endoscopic treatment of unilateral primary haematuria: Is non-specific diffuse pyelitis a real entity? Eur Urol 1994;26:109-114.

8. Nakada SY, Elashry OM, Picus D, Clayman RV. Longterm outcome of flexible ureterorenoscopy in the diagnosis and treatment of lateralizing essential hematuria. J Urol 1997; 157:776-779.

9. Tawfiek ER, Bagley DH. Ureteroscopic evaluation and treatment of chronic unilateral hematuria. J Urol 1998;160 (3 Pt 1):700-702.

10. Mugiya S, Ohhira T, Un-No T, Takayama T, Suzuki K, Fujita K. Endoscopic management of upper urinary tract disease using a 200-microm holmium laser fiber: Initial experience in Japan. Urology 1999;53:60-64.

11. Yazaki T, Kamiyama Y, Tomomasa H, et al. Ureteropyeloscopy in the diagnosis of patients with upper tract hematuria: An initial clinical study. Int J Urol 1999;6:219-225.

12. Mugiya S, Ozono S, Nagata M, Takayama T, Furuse H, Ushiyama T. Ureteroscopic evaluation and laser treatment of chronic unilateral hematuria. J Urol 2007;178:517-520.

13. Brito AH, Mazzucchi E, Vicentini FC, Danilovic A, Chedid Neto EA, Srougi M. Management of chronic unilateral hematuria by ureterorenoscopy. J Endourol 2009;23:1273-1276.

14. Araki M, Uehara S, Sasaki K, et al. Ureteroscopic management of chronic unilateral hematuria: A single-center experience over 22 years. PLoS One 2012;7:e36729.

15. Takazawa R, Kitayama S, Tsujii T. Digital ureteroscopic visualization of lesions responsible for chronic unilateral hematuria, so-called idiopathic renal bleeding. Int J Urol 2014;21:227-228.

16. Hagen A. Renal angioma. Four cases of angioma of the renal pelvis. Acta chir Scand 1963;126:657-667.

17. Rowbotham C, Anson KM. Benign lateralizing haematuria: The impact of upper tract endoscopy. BJU Int 2001;88:841849.

18. Diamond DA, Jeffs RD, Marshall FF. Control of prolonged, benign, renal hematuria by silver nitrate instillation. Urology 1981;18:337-341.

19. Vijan SR, Keating MA, Althausen AF. Ureteral stenosis after silver nitrate instillation in the treatment of essential hematuria. J Urol 1988;139:1015-1016.

20. Stefanini M, English HA, Taylor AE. Safe and effective, prolonged administration of epsilon aminocaproic acid in bleeding from the urinary tract. J Urol 1990;143:559-561.
21. Manjunath G, Fozailoff A, Mitcheson D, Sarnak MJ. Epsilon-aminocaproic acid and renal complications: Case report and review of the literature. Clin Nephrol 2002;58: 63-67.

22. Kaye JD, Smith EA, Kirsch AJ, Cerwinka WH, Elmore JM. Preliminary experience with epsilon aminocaproic acid for treatment of intractable upper tract hematuria in children with hematological disorders. J Urol 2010;184:1152-1157.

23. Nash DA, Jr., Henry AR. Unilateral essential hematuria. Therapy with epsilon aminocaproic acid. Urology 1984;23:297-298.

24. Lano MD, Wagoner RD, Leary FJ. Unilateral essential hematuria. Mayo Clin Proc 1979;54:88-90.

25. Bagley DH, Tanimoto R, Healy KA. Upper tract urothelial carcinoma: Ureteroscopic biopsy and specimen preparation. Upper Uninary Tract Urothelial Carcinoma 2015; Springer:1-16.

26. Abdel-Razzak OM, Ehya H, Cubler-Goodman A, Bagley $\mathrm{DH}$. Ureteroscopic biopsy in the upper urinary tract. Urology 1994;44:451-457.

27. Tawfiek E, Bibbo M, Bagley DH. Ureteroscopic biopsy: Technique and specimen preparation. Urology 1997;50: 117-119.

28. Jinzaki M, Matsumoto K, Kikuchi E, et al. Comparison of CT urography and excretory urography in the detection and localization of urothelial carcinoma of the upper urinary tract. AJR Am J Roentgenol 2011;196:1102-1109.

29. Wang LJ, Wong YC, Huang CC, Wu CH, Hung SC, Chen HW. Multidetector computerized tomography urography is more accurate than excretory urography for diagnosing transitional cell carcinoma of the upper urinary tract in adults with hematuria. J Urol 2010;183:48-55.

30. Albani JM, Ciaschini MW, Streem SB, Herts BR, Angermeier $\mathrm{KW}$. The role of computerized tomographic urography in the initial evaluation of hematuria. J Urol 2007; 177:644-648.

31. Gray Sears CL, Ward JF, Sears ST, Puckett MF, Kane CJ, Amling CL. Prospective comparison of computerized tomography and excretory urography in the initial evaluation of asymptomatic microhematuria. J Urol 2002;168:2457-2460.

\section{Address correspondence to: Ryuta Tanimoto, $\mathrm{MD}, \mathrm{PhD}$ Department of Urology Okayama University Hospital 2-5-1 Shikata-cho Okayama 700-8558 Japan}

E-mail: tanimo10@gmail.com

$\begin{aligned} & \text { Abbreviations Used } \\ & \mathrm{CCD}=\text { charge-coupled device } \\ & \mathrm{CMOS}=\text { complementary metal-oxide-semiconductor } \\ & \mathrm{CT}=\text { computed tomography } \\ & \mathrm{CTU}=\text { computed tomography urogram } \\ & \mathrm{CUH}=\text { chronic unilateral hematuria } \\ & \mathrm{FU}=\text { flexible ureteroscope } \\ & \mathrm{IVU}=\text { intravenous urogram } \\ & \mathrm{MVR}=\text { minute venous rupture } \\ & \mathrm{YAG}=\text { yttrium aluminium garnet }\end{aligned}$

